Wu et al., Afr J Tradit Complement Altern Med., (2017) 14 (4): 201-212

https://doi.org/10.21010/ajtcam.v14i4.23.

\title{
ANTIOXIDANT AND ANTI-INFLAMMATORY EFFECTS OF RHAMNAZIN ON LIPOPOLYSACCHARIDE-INDUCED ACUTE LUNG INJURY AND INFLAMMATION IN RATS
}

\author{
GuoRong Wu ${ }^{1}$, XiaoPing Dai ${ }^{1}$, XiangRong Li ${ }^{1}$, HePing Jiang ${ }^{1 *}$ \\ ${ }^{1}$ Department of respiratory medicine, Changzhou Jintan District People's Hospital, Changzhou, Jiangsu 213200, \\ China
}

*Corresponding Author E-mail: jiangheping77@ @otmail.com

\begin{abstract}
Background: Acute Lung Injury (ALI) results into severe inflammation and oxidative stress to the pulmonary tissue. Rhamnazin is a natural flavonoid and known for its antioxidant and anti-inflammatory properties.

Materials and methods: The antioxidative and anti-inflammatory properties rhamnazin were tested for protection against the acute lung injury. We investigated whether rhamnazin improves the lipopolysaccharide (LPS)-induced ALI in an animal model (rat). We also studied the probable molecular mechanism of action of rhamnazin. Rhamnazin was injected intraperitoneally (i.p.) $(5,10$ and $20 \mathrm{mg} / \mathrm{kg}$ ) two days before intratracheal LPS challenge $(5 \mathrm{mg} / \mathrm{kg})$. The changes in lung wet-to-dry weight ratio, LDH activity, pulmonary histopathology, BALF protein concentration, MPO activity, oxidative stress, cytokine production were estimated.

Results: The results showed a significant attenuation of all the inflammatory parameters and a marked improvement in the pulmonary histopathology in the animal groups pretreated with rhamnazin. The rhamnazin pretreated group also showed activation of Nrf2 pathway and attenuation of ROS such as $\mathrm{H}_{2} \mathrm{O}_{2}$, MDA and hydroxyl ion. These results indicated that rhamnazin could attenuate the symptoms of ALI in rats due to its strong antioxidant and anti-inflammatory properties.

Conclusion: The results strongly demonstrated that rhamnazin provides protection against LPS-induced ALI. The underlying mechanisms of its anti-inflammatory action may include inhibition of Nrf2 mediated antioxidative pathway.
\end{abstract}

Keywords: acute lung injury, inflammation, cytokine, BALF, flavonoid

\section{Introduction}

Acute lung injury (ALI) is a severe form of respiratory failure syndrome. ALI is characterized by pulmonary edema, acute respiratory failure and capillary edema (Luh and Chiang, 2007). These symptoms make ALI a life-threatening disorder resulting in high mortality in patients (Ware and Matthay, 2000; Frutos-Vivar et al; 2004). The pathogenesis of this disease includes acute and chronic inflammation, causing elevated permeability of pulmonary vessels. This increased permeability leads to pulmonary edema and infiltration of cells into the alveolar cavities causing the destruction of respiration (Piantadosi and Schwartz, 2004).

ALI may result due to several causes such as aspiration, toxic inhalation, and drowning. ALI may also be caused due to several indirect cases such as burns, blood transfusion, sepsis etc. All these causes result in pulmonary inflammation which is caused due to the response of vascular tissues towards, the above-stated causes. Gram-negative bacteria have been recognized as the main cause of this infection. Lipopolysaccharide (LPS), present in the bacterial cell wall is an important virulence factor and is a primary factor known to cause the pulmonary inflammation (Ingenito et al; 2001, Karmpaliotis et al; 2002). LPS has been reported to cause ALI through several inflammatory mechanisms (Sanlioglu et al; 2001;Sato et al; 2002; Suliman et al; 2003). LPS induces a pro-inflammatory tumor necrosis factor (TNF) dependent pathway and causes an increased expression of cytokines (Tang et al; 2001; Liu and Lin, 2007). These cytokines include IL-1 $\beta$, including interleukin (IL)-6, and macrophage inflammatory protein 2 (MIP-2), leading to amplification of inflammation signal (Tang et al; 2001; Liu and Lin, 2007). The In addition to activation the generation of cytokines is also induced upon LPS exposure. These cytokines include TNF- $\alpha$ and IL-6. These cytokines are known to further activate a feedback loop, thus amplifying the pro-inflammatory signal (Ware and Matthay, 2000; Li and Verma, 2002).

Oxidative stress and inflammation are closely linked and have an important role in the pathogenesis of ALI induced by LPS. Oxidative stress causes recruitment of leucocytes and other resident cells leading to inflammation 
Wu et al., Afr J Tradit Complement Altern Med., (2017) 14 (4): 201-212

https://doi.org/10.21010/ajtcam.v14i4.23.

(Cachofeiro et al; 2008). Conversely, these cells cause induction of oxidative stress by ROS, nitrogen and chlorine species. Nuclear factor erythroid-2-related factor 2 (Nrf2) is a transcription factor responsible for induction of genes related to antioxidant response elements (Pedruzzi et al; 2012). The activation of Nrf2 is used by the host cells in response to such oxidative stresses. Nrf2 is also known to function as a master regulator of the antioxidant response genes (Deramaudt et al; 2013).

Flavonoids are a group of natural substances known for their several anti-inflammatory and antioxidative properties (Nijveldt et al; 2001). Some flavonoids have previously been indicated for their role in ALI for example, Quercetin (Huang et al; 2015) and Naringenin (Fouad et al; 2016). Rhamnazin is a natural flavonoid found in plants such as the blackthorn plant. Rhamnazin has been shown to possess strong antioxidant and anti-inflammatory properties (Martini et al; 2004). However, no study has yet shown its function in acute lung injury induced by LPS. In the present study, we explored, whether rhamnazin can potentially protect against LPS-induced ALI. Additionally, we also elucidated its potential molecular mechanism of action.

\section{Materials and methods}

Study animals and design of experiment

Sprague-Dawley rats (adult males, $\mathrm{n}=60$, weighing 250 to $300 \mathrm{~g}$ ) were provided by the animal house of Changzhou jintan district people's hospital. The animals were kept under a $12 \mathrm{~h}$ by $12 \mathrm{~h}$ light and dark circadian cycle and the under controlled conditions of temperature and humidity. The animals were fed ad libitum, with the standard rat diet and water. All the animals' up-keeping procedures were carried out with approval from the institutional ethical committee and in accordance with the Principles of Laboratory Animal Care (NIH publication 85-23, revised in 1985). All efforts were made to minimize the number of animals and their suffering.

Rhamnazin and Escherichia coli LPS were procured from Sigma (USA). The animals were assigned to six groups of 10 rats each at random viz. 1) Control (phosphate buffered saline); 2) LPS group (5 mg/kg, administered through intratracheal instillation); (3) LPS + vehicle; 4) LPS+Rhamnazin (5 mg/kg) group; (5) LPS+ Rhamnazin (10 mg/kg) group; (6) LPS + Rhamnazin $(20 \mathrm{mg} / \mathrm{kg}$ ) group. Saline and Rhamnazin were intra-peritoneally (i.p.) administered $48 \mathrm{~h}$ before the LPS challenge. The rats were euthanized at 24 hours post-LPS administration. The euthanasia was followed by the collection of lung samples for subsequent experimental analysis.

\section{Estimation of wet-to-dry weight ratio of the lungs}

The extent of pulmonary edema in the experimental groups was assessed by measuring the wet-to-dry weight of the lungs (Yang et al; 2005). For this, the left lung was excised immediately, rinsed in phosphate buffer saline (PBS) and weighed to determine the lung 'wet' weight. The lung was then desiccated for $48 \mathrm{~h}$ at $85^{\circ} \mathrm{C}$ temperature in an oven. The lung was again weighted to estimate its 'dry' weight. The ratio of the wet-to-dry weight of lung was calculated to quantify pulmonary edema.

\section{Lactate dehydrogenase (LDH) assay}

The activity of LDH, an enzyme used as a marker for cytotoxicity, was measured as decribed previously (Lee et al; 2015). LDH activity was measured using LDH estimation kit (Sigma, USA). The supernatants of BALF was added to the reagent A ( $2 \mathrm{ml})$ for $60 \mathrm{~s}$ and then the reagent B was added. Subsequently, the recording of absorbance was done for $4 \mathrm{~m}$ at every 60s. The activity of LDH was recorded in U/l. The LDH standards provided by sigma kit were used to ascertain the linearity of the curve.

\section{Estimation of the broncho-alveolar lavage (BAL) protein concentration and the cell counts}

The protein concentration in BALF was performed as described by Lee et al. (2010). Briefly, Broncho-alveolar lavage (BAL) was done by lavaging with $5 \mathrm{ml}$ of PBS and a gentle aspiration. Approximately $90 \%$ of the BALF was recovered by lavaging. Centrifugation of the BALF was carried out at $1000 \mathrm{~g}$ for 30 minutes at $4{ }^{\circ} \mathrm{C}$ temperature. The estimation of total protein in the supernatants was performed by Bradford's method and using the Protein Assay Dye (BioRad, USA). Protein estimation was performed by generating a standard curve of bovine serum albumin protein (BSA). Moreover, estimation of total cell count in the BALF was carried out post re-suspension of the cell pellet in $50 \mu 1$ PBS. 
Wu et al., Afr J Tradit Complement Altern Med., (2017) 14 (4): 201-212

https://doi.org/10.21010/ajtcam.v14i4.23.

\section{Estimation of pulmonary Myeloperoxidase (MPO) activity}

ALI is characterized by increased neutrophil accumulation in lungs. This elevated neutrophil accumulation can be detected by the MPO activity (Kim et al. 2012). For estimation of MPO activity, equal weights of the lung tissues were homogenized and centrifuged at $15000 \mathrm{~g}$ for 30 minutes at $4{ }^{\circ} \mathrm{C}$ temperature. The supernatant thus separated was evaluated for MPO activity using the MPO ELISA kit (Hycult Biotech, Netherlands) as per the manufacturer's protocol and absorbance was measured at $450 \mathrm{~nm}$. The absorbance of the samples was measured at $450 \mathrm{~nm}$ wavelength. All the evaluations were performed in replicates and the units were depicted in per gram.

\section{Measurement of the pulmonary oxidative stress}

Oxidative stress parameters were measured as described previously (Trocha et al. 2014). Briefly, the excised lungs were homogenized at $10 \%(\mathrm{w} / \mathrm{v})$ and subsequently centrifuged at $15000 \mathrm{~g}$ and $4{ }^{\circ} \mathrm{C}$ temperature for 30 minutes and the supernatant thus obtained was used for oxidative stress evaluations using colorimetric methods (spectrophotometer MARCEL S350 PRO). The activity of Hydrogen peroxide $\left(\mathrm{H}_{2} \mathrm{O}_{2}\right)$, Malondialdehyde (MDA) and Hydroxyl radical $(\mathrm{OH})$ was estimated using the Hydrogen peroxide assay kit (Abcam, USA), lipid peroxidation (MDA) assay kit (Abcam, USA) and hydroxyl radical antioxidant capacity assay kit (Eagle, bioscience, USA) respectively.

\section{Examination of pulmonary histopathology}

Histopathological examination was performed as described by Li et al. (2016). The upper lobes of the right lungs were excised from all the six experimental groups. The lung tissues were fixed in $8 \%$ paraformaldehyde for 24 hours, dehydrated in ethanol and sliced into $4 \mu \mathrm{m}$ sections after embedding them in paraffin. Post sectioning, the tissues were deparaffinized and subsequently stained using the hemotoxylin-eosin (H\&E) stain. Histopathological variations in lungs were analyzed under a light microscope.

\section{Cytokine measurements}

The concentrations of cytokines (IL-6 and TNF- $\alpha$ ) in the serum and BALF were estimated using ELISA kit (MN, USA), following the manufacturer's protocol.

\section{Western Blot analysis}

Antibodies for Nrf2, Trx1, and $\beta$-actin were procured from the Abcam Inc. (USA). The concentrations of the proteins were estimated as mentioned above. $15 \mu \mathrm{g}$ of the estimated protein was loaded per well on $12 \%$ a sodium dodecyl sulfate polyacrylamide (SDS-PAGE) gel. Subsequently, the proteins were electrophoretically transferred on a polyvinylidene difluoride (PVDF) membrane after completion of gel run. Blocking of the membranes was performed for 1.5 hours in Tris-buffered saline which contained $0.2 \%$ Tween 20 (TBST) and $2 \%$ non-fat dry milk (NFDM). Binding of primary antibodies onto the membranes was performed by incubating it with primary antibody solution in TBST and $2 \%$ NFDM for 8 hours at $4{ }^{\circ} \mathrm{C}$ temperature. Then the membrane washing was performed three times with TBST. Subsequently, the membranes were incubated with goat anti-mouse secondary antibodies for $2 \mathrm{~h}$. Finally, the membranes incubated for 1 hour with horse radish peroxidase (HRP) conjugated secondary antibodies and developed using an ECL detection system. The band density on the membranes was scanned and then analyzed using the ImageJ (NIH, USA) software program.

\section{Isolation of total RNA and qRT-PCR}

Total RNA was isolated from macrophages using the RNeasy Mini Kit (Qiagen, USA) subsequently qRT-PCR was performed using One-Step qRT-PCR Kit (Qiagen, USA) following the manufacture's protocol. The designing of qRTPCR primers was done using the primer blast (Ye et al. 2012). The melting temperature (Tm) of primers was taken between $57^{\circ} \mathrm{C}$ and $63^{\circ} \mathrm{C}$. The size of the amplification product was taken between 90 and $120 \mathrm{bp}$ along with a difference in $\mathrm{Tm}$ of $3^{\circ} \mathrm{C}(\max )$. We took three technical replicates for every biological replicate. The quality of RNA was assessed by a QIAxpert ${ }^{\mathrm{TM}}$ microfluidic UV/VIS spectrophotometer (Qiagen, USA). The PCR master mix included $5 \mu$ l DyNAmo Flash SYBR Green (Thermo) (2X), $1.5 \mu \mathrm{l} \mathrm{cDNA}, 1 \mu \mathrm{l}(5 \mathrm{pm} / \mu \mathrm{l})$ each primer. The following cycling conditions were used for qRT-PCR: denaturation at $95^{\circ} \mathrm{C}$ for $10 \mathrm{~min}, 40$ denaturation cycles at $95^{\circ} \mathrm{C}$ for $22 \mathrm{~s}$, annealing with extension at $60^{\circ} \mathrm{C}$ for 
Wu et al., Afr J Tradit Complement Altern Med., (2017) 14 (4): 201-212

https://doi.org/10.21010/ajtcam.v14i4.23.

60 s. The qRT-PCR amplification was performed in ABI 7500 system (Applied Biosystem). The threshold cycle value (Ct) for the genes were quantified and normalized by Ct value Glyceraldehyde-3-Phosphate Dehydrogenase (GADPH)

expression. The relative expression was calculated using the $2^{-\Delta \Delta C t}$ method (Livak and Schmittgen 2001). The following

primers were used for qRT-PCR: mouse Nrf2 (5'- CAGAGTGATGGTTGCCCACT-3', 5'-

CACACACTTTCTGCGTGCTC-3'), Trx1 (5'-AAAGGGTCAAAAGGTGGGGG-3', 5'-

ACAGCTGGTAGCTGGTTACAC-3'); IL-6 (5'-CCAGAATACTTCGAAGTTCC-3', 5'-TATCGGTTAATCCCAAG-3'),

TNF- $\alpha,\left(5^{\prime}-\right.$ GATCGGTCCCCAAAGGGATG-3', 5'-CCACTTGGTGGTTTGTGAGTG-3'), MIP-2 (5'-

GCTGTCCCTCAACGGAAGAA-3', 5'-CTCAGACAGCGAGGCACATC-3'), IL-1 $\beta$ (5'-

GCCACCTTTTGACAGTGATGAG-3', 5'-GACAGCCCAGGTCAAAGGTT-3'), GAPDH (5'-

GAGAGAGGCCCAGCTACTCG-3', 5'-CCTCCCGCCCTGCTTATCC-3') was taken as an internal control.

\section{Statistical evaluation}

The quantitative results were depicted as means \pm SEM. All the quantitative results were statistically analyzed by one-way analysis of variance (ANOVA). The analysis for multiple tests correction was performed by Bonferroni's method. All the statistical calculations were performed in SPSS software program. The maximum threshold for statistically significant $\mathrm{P}$-values was set at 0.05 .

\section{Results}

\section{The effect of rhamnazin on pulmonary hyperpermeability and edema}

Exposure to LPS caused a significant increase in the wet-to-dry weight ratio of the lungs (Fig. 1) when compared to the control treated and vehicle treated animals. Additionally, the BALF protein concentration also showed a significant increase (Fig. 1) in comparison to the control treated and vehicle treated animals. These two parameters were significantly reduced in rhamnazin pre-treatment groups in a dose dependant manner. Similarly, the levels of LDH were significantly increased in the LPS treated animals. The LDH levels were significantly reduced upon treatment with rhamnazin in a dose dependant manner.

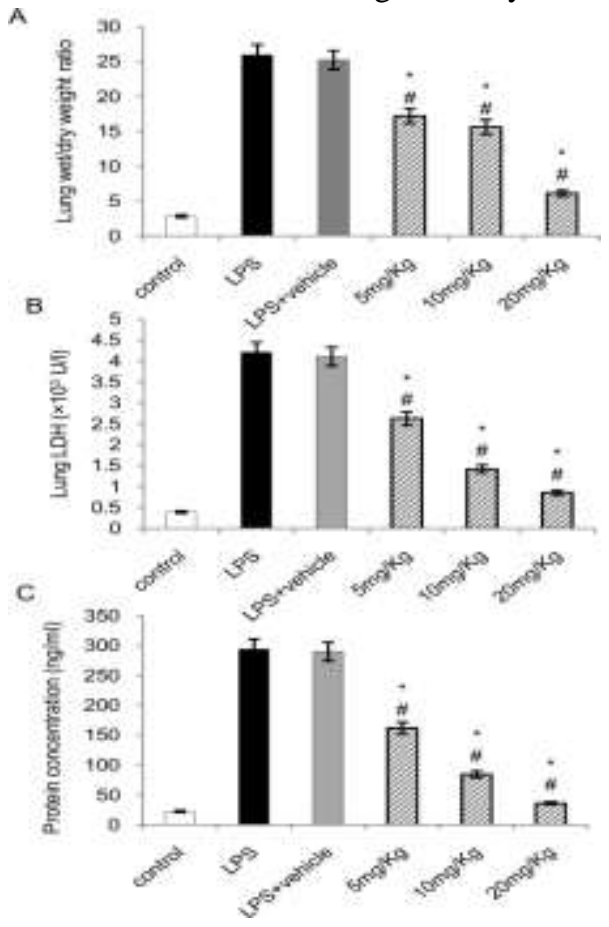

Figure 1: Effect of rhamnazin pretreatment on (A) lung wet/dry ratio, (B) lung tissue LDH level and (C) protein concentration in the bronchoalveolar lavage fluid. Data are presented as the mean \pm standard error $(\mathrm{n}=10)$. $* \mathrm{P}<0.05$ compared with the LPS-induced ALI group; \#P<0.05 compared with the vehicle-treated ALI group. ALI, acute lung injury; LPS, lipopolysaccharide; LDH, lactate dehydrogenase.

Effect of rhamnazin on the histopathologic variations 
Wu et al., Afr J Tradit Complement Altern Med., (2017) 14 (4): 201-212

https://doi.org/10.21010/ajtcam.v14i4.23.

Histological examination of the lung tissue was performed 24 hours after LPS induction. As depicted in the Fig. 2, the lung tissues of the control treated and vehicle treated animal groups had a normal appearance and were devoid of any histopathological changes. It was observed that exposure to LPS treatment exhibited extensive damage to the lung tissue. The large-scale intrusion of the inflammatory cells inside the pulmonary interstitium along with migration into the alveolar cavities. Moreover, the LPS treatment also resulted into pulmonary edema, hemorrhagia in the stroma, alveolar collapse, and alveolar wall thickening. The LPS group that was treated with rhamnazin showed a significant attenuation of the histopathological changes especially in the group of animals that were treated with $10 \mathrm{mg}$ and $20 \mathrm{mg} / \mathrm{kg}$ of rhamnazin.
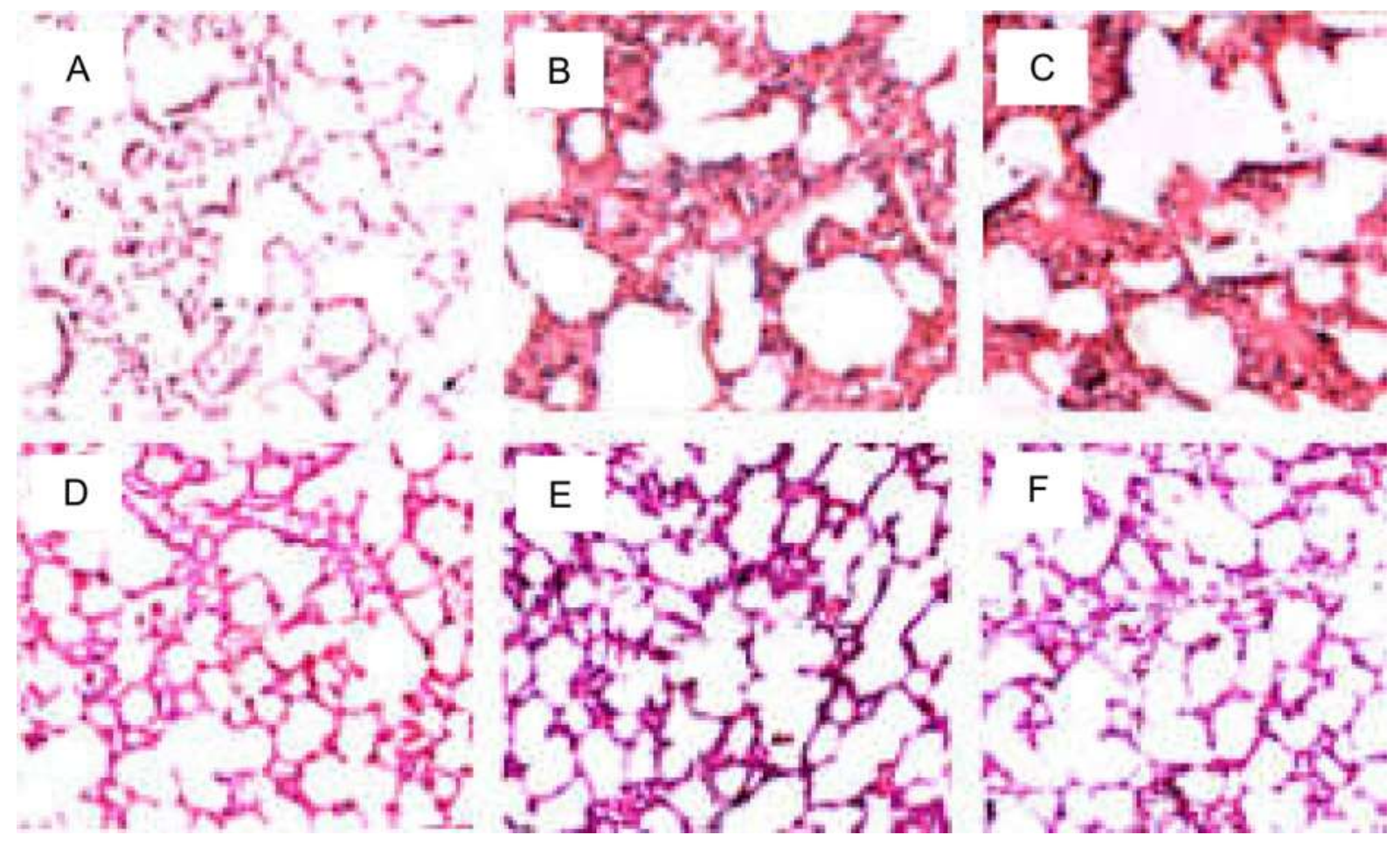

Figure 2: Effect of rhamnazin pretreatment on pulmonary histopathology. (A) phosphate-buffered saline-treated healthy control group, (B) LPS-induced ALI group, (C) vehicle-treated ALI group and (D) $5 \mathrm{mg} / \mathrm{kg}$ rhamnazin pretreated ALI group (E) $10 \mathrm{mg} / \mathrm{kg}$ rhamnazin pretreated ALI group, and (D) $20 \mathrm{mg} / \mathrm{kg}$ rhamnazin pretreated ALI group.

\section{Effect of rhamnazin treatment on production of cytokines}

The levels of pro-inflammatory cytokines viz. IL-6, TNF- $\alpha$, MIP-2, and IL-1 $\beta$ in BALF and serum of different experimental groups were measured using ELISA. LPS induction caused a significant increase in the serum levels of these cytokines in serum (Fig. 3) as well as BALF (Fig. 4). Pretreatment with rhamnazin had a significant effect on the levels of cytokines both in serum and BALF when compared to control treated and vehicle treated animal groups. The rhamnazin treatment groups showed a significant reduction in the levels of IL-6, TNF- $\alpha$, MIP-2, and IL-1 $\beta$, both in serum and BALF. 
Wu et al., Afr J Tradit Complement Altern Med., (2017) 14 (4): 201-212

https://doi.org/10.21010/ajtcam.v14i4.23.

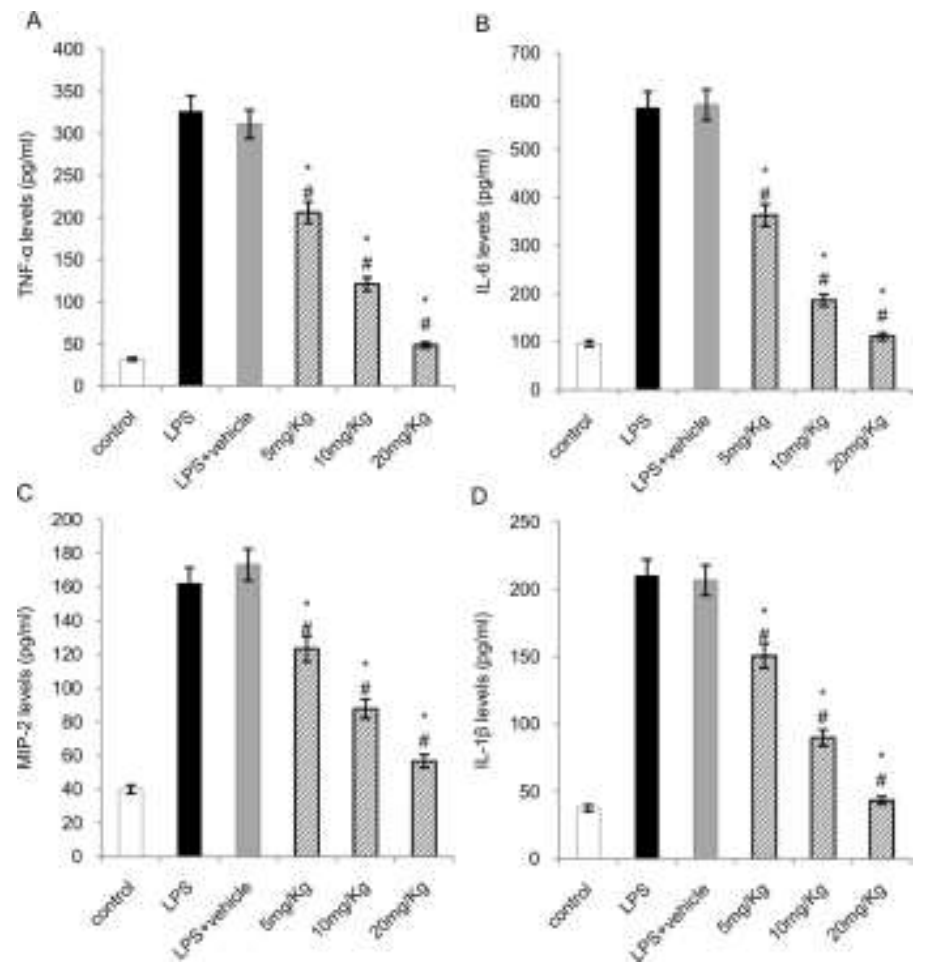

Figure 3: Effect of rhamnazin pretreatment on LPS-induced pro-inflammatory cytokine secretion in the serum of differentially treated ALI mice. Levels of (A) TNF- $\alpha$, (B) IL-6, (C) MIP-2 and (D) IL-1 $\beta$ in the serum.

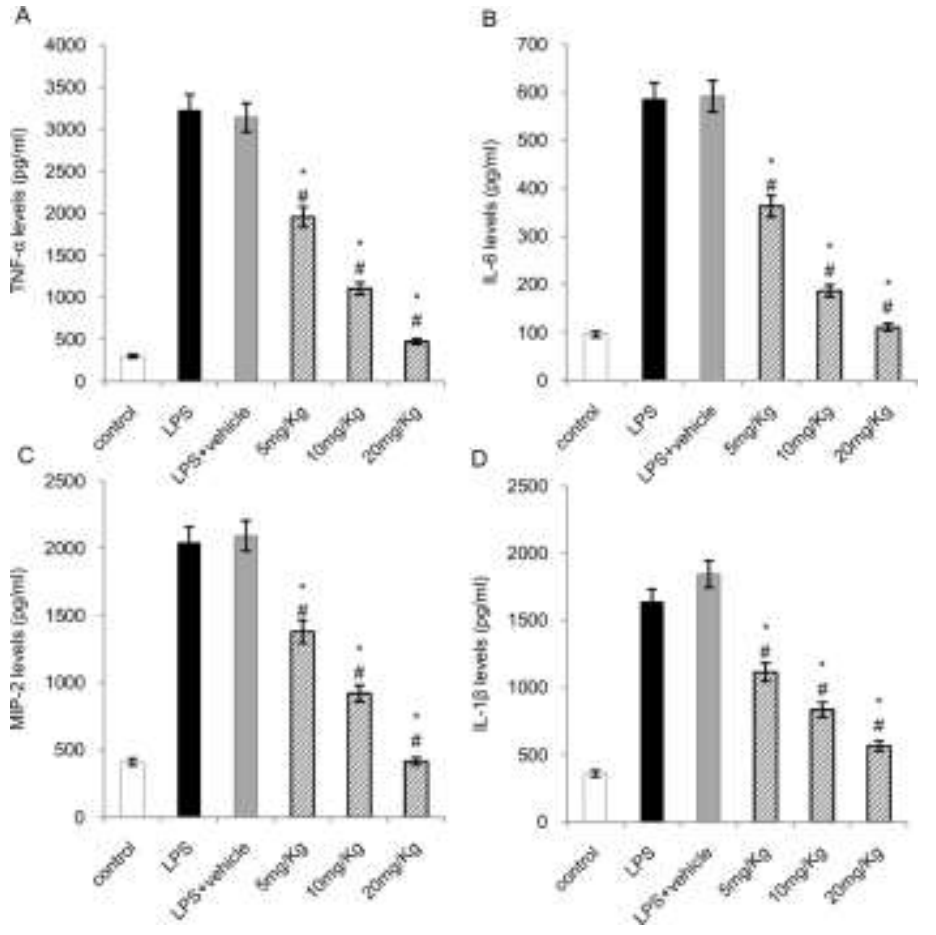

Figure 4: Effect of rhamnazin on LPS-induced pro-inflammatory cytokine secretion in the BALF of differentially treated ALI mice. Levels of (A) TNF- $\alpha$, (B) IL-6, (C) MIP-2 and (D) IL-1 $\beta$ in the BALF. 
Wu et al., Afr J Tradit Complement Altern Med., (2017) 14 (4): 201-212

https://doi.org/10.21010/ajtcam.v14i4.23.

\section{Effect of rhamnazin on MPO activity}

The MPO activity and neutrophil count was estimated in homogenates of pulmonary tissue. Moreover, the count of neutrophils in BALF was also estimated in the different experimental groups. Induction with LPS caused a significant increase in the activity of MPO (Fig. 5). Similarly, the neutrophil count also showed a significant increase in the BALF of LPS induced animals. Upon pretreatment with rhamnazin, the increased activity of MPO as well as increased neutrophil number showed a significant decrease in comparison to control treated and vehicle treated animal groups in a dose dependant manner. However, the $5 \mathrm{mg} / \mathrm{kg}$ group showed no significant attenuation of MPO activity.

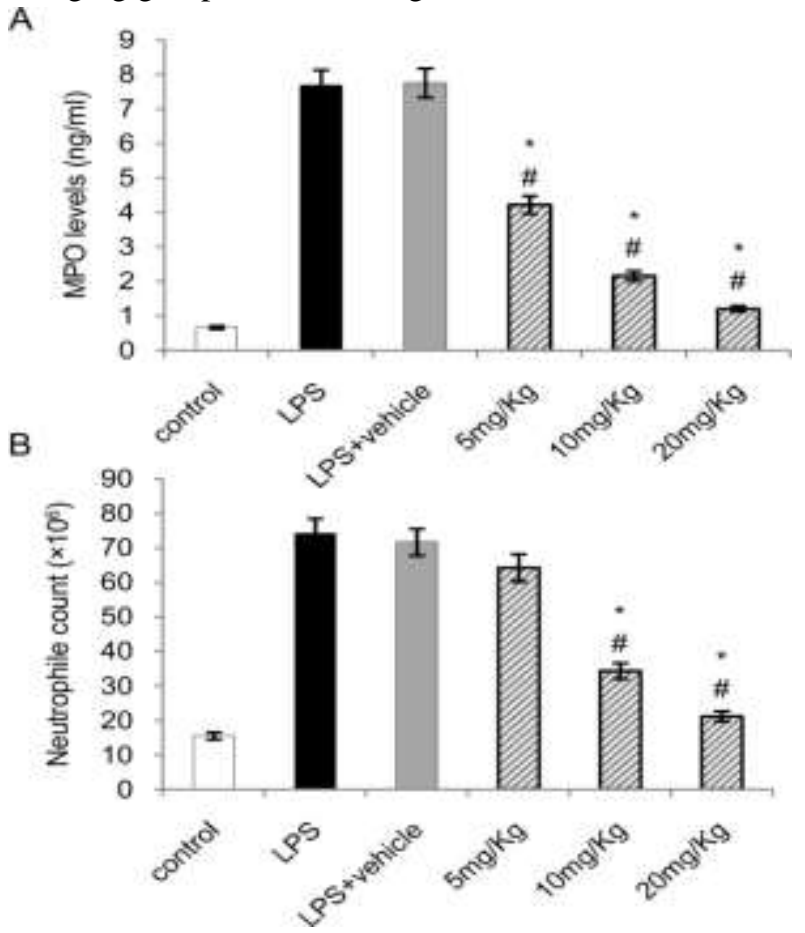

Figure 5: Effect of rhamnazin pretreatment on MPO (myeloperoxidase) activity and neutrophil accumulation in lung tissues. (A) MPO activity in the lung tissue homogenates of differentially treated ALI mice. (B) Neutrophil count in the lung bronchoalveolar lavage fluid of differentially treated ALI mice. Data are presented as the mean \pm standard error $(\mathrm{n}=10)$. $* \mathrm{P}<0.05$ compared with the LPS-induced ALI group; $\# \mathrm{P}<0.05$ compared with the vehicle-treated ALI mice.

\section{Effects of rhamnazin on oxidative stress levels of ALI animals}

The levels of oxidative stress in LPS induced ALI rats was estimated by determining the concentration of MDA, $\mathrm{H}_{2} \mathrm{O}_{2}$ and hydroxyl ion in the experimental groups in vivo. The induction of ALI caused a significant increase in the levels of MDA, $\mathrm{H}_{2} \mathrm{O}_{2}$ and hydroxyl ion. Upon treatment with rhamnazin at different concentrations, a significant decrease in the levels of oxidative stress was observed in the $10 \mathrm{mg} / \mathrm{kg}$ and $20 \mathrm{mg} / \mathrm{kg}$ treatment groups (Fig. 6). These results showed that rhamnazin has can potentially attenuate the oxidative stress levels in ALI animals induced with LPS. However, the $5 \mathrm{mg} / \mathrm{kg}$ group showed no significant attenuation of oxidative stress. 
Wu et al., Afr J Tradit Complement Altern Med., (2017) 14 (4): 201-212

https://doi.org/10.21010/ajtcam.v14i4.23.

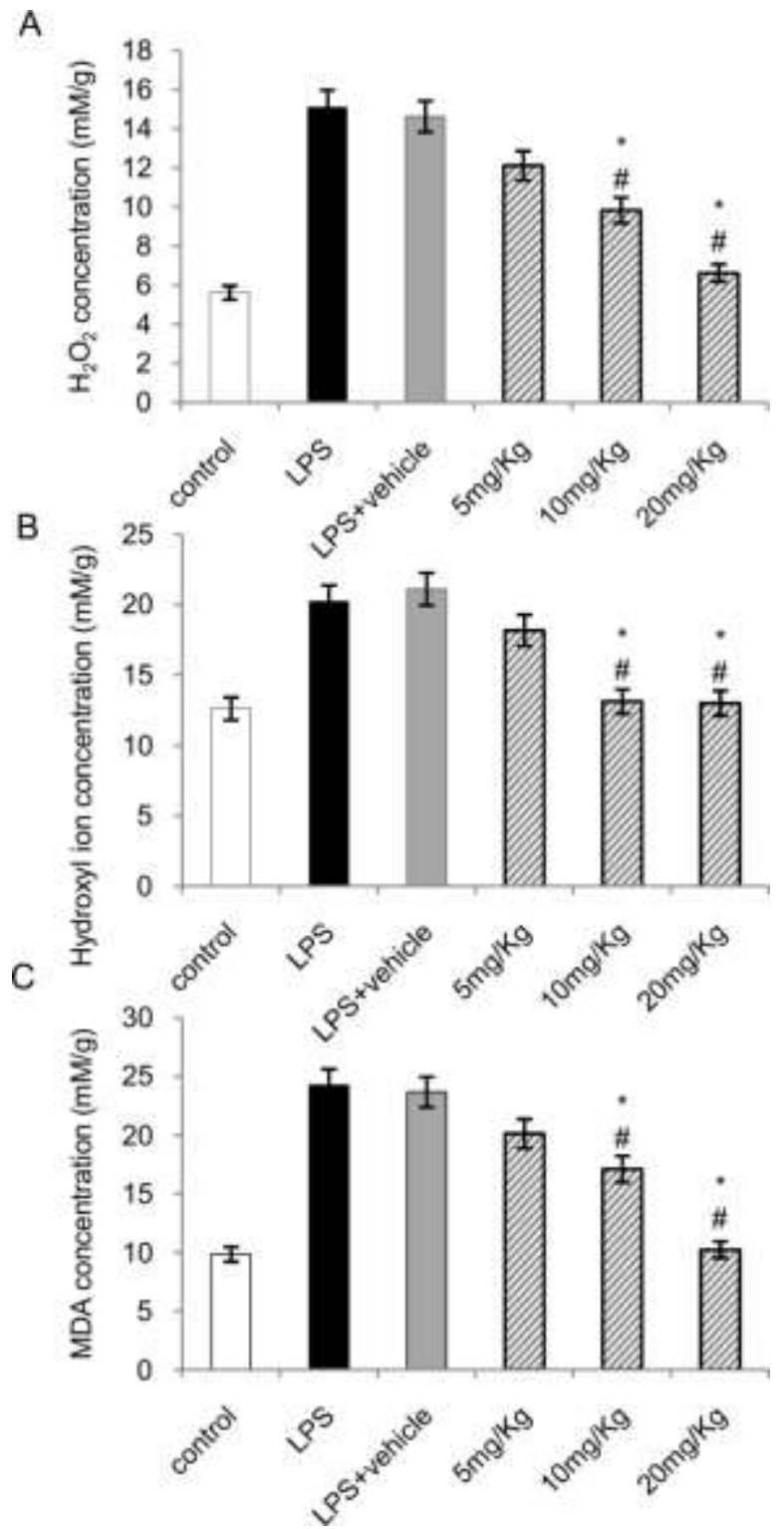

Figure 6: Effect of rhamnazin pretreatment on oxidative stress. The levels of (A) $\mathrm{H}_{2} \mathrm{O}_{2}$, (B) hydroxyl ion and (C) MDA in the homogenized lung tissue. Data are presented as the mean \pm standard error $(n=10)$. ${ }^{*} \mathrm{P}<0.05$ compared with the LPSinduced ALI group; \#P<0.05 compared with the vehicle-treated ALI group. MDA, malondialdehyde.

\section{Effects of rhamnazin treatment on the levels of Nrf1 and Trx1}

To estimate the antioxidant response of pulmonary cells, the levels of Nrf1 and Trx 1 were estimated using qRTPCR and western blot analysis. qRT-PCR results showed that as compared to control group, LPS induced ALI animals had a significantly decreased expression of Nrf1 and Trx1 mRNA (Fig. 7). Whereas, the pretreatment with rhamnazin caused a significant increase in the levels of mRNA of Nrf1 and Trx1. The results of western blot analysis also showed a similar trend as that of mRNA expression levels (Fig. 8). In western blot, the protein expression levels of Nrf1 and Trx1 were significantly reduced in ALI animals as compared to the control treated and vehicle treated groups. Whereas, the rhamnazin pretreated group showed significantly elevated levels of the two proteins in a dose dependant manner. 
Wu et al., Afr J Tradit Complement Altern Med., (2017) 14 (4): 201-212

https://doi.org/10.21010/ajtcam.v14i4.23.

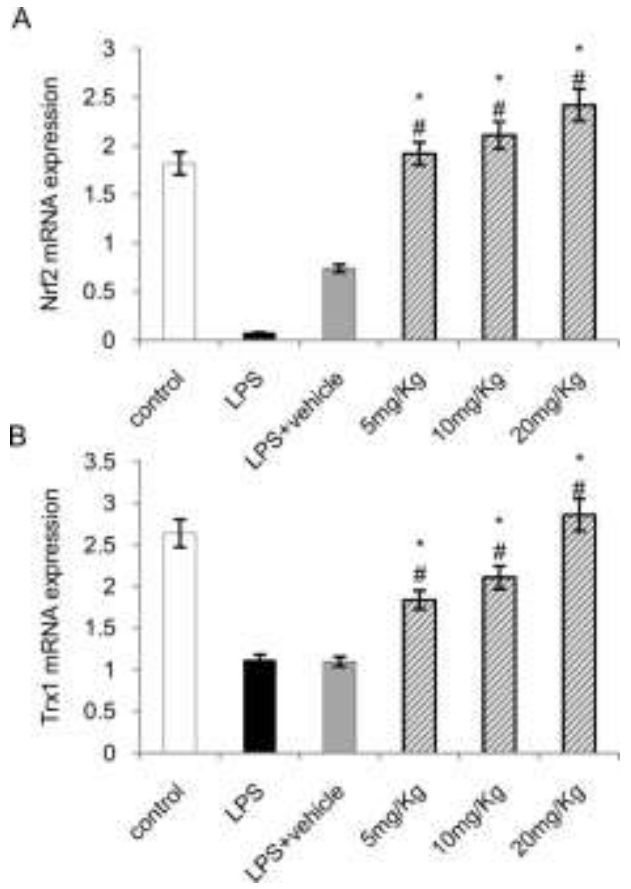

Figure 7: Effect of rhamnazin pretreatment on Nrf2 and Trx1 expression in lung tissues. The mRNA expression levels of (A) Nrf2 and (B) Trx1 are shown. Data are presented as the mean \pm standard error $(n=10)$. $* P<0.05$ compared with the LPS-induced ALI group; \#P $<0.05$ compared with the vehicle-treated ALI group. Nrf2, nuclear factor erythroid-2-related factor 2; Trx1, thioredoxin isoform 1 .

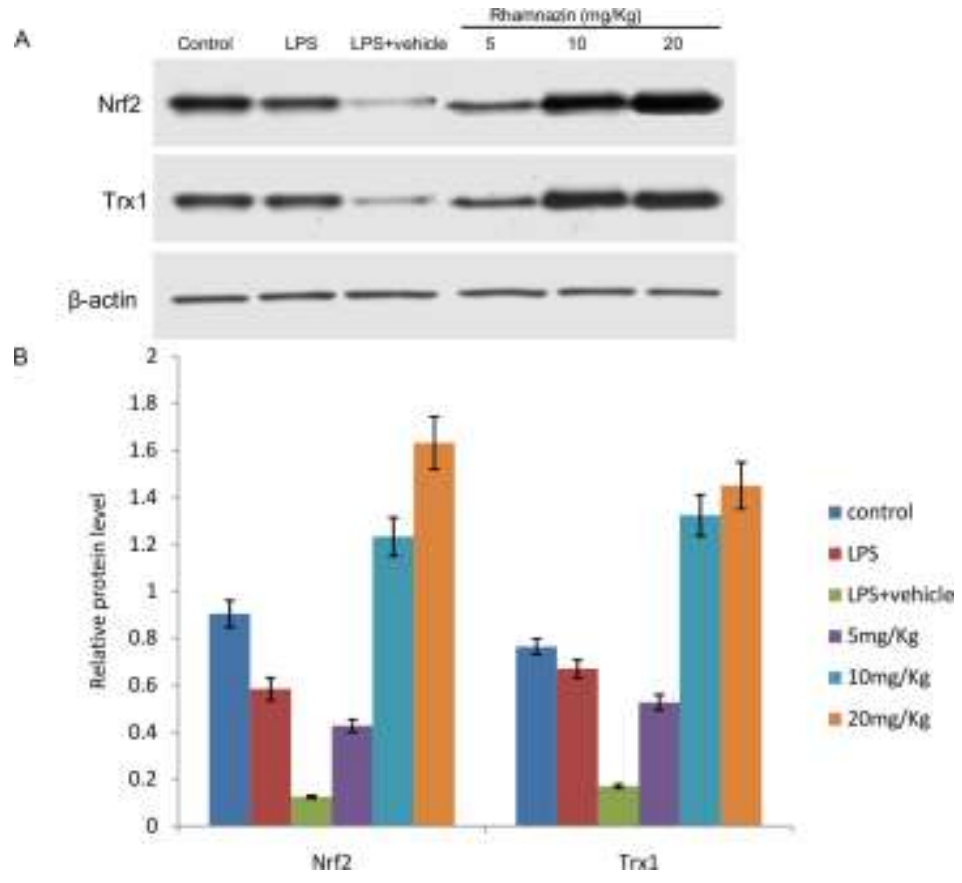

Figure 8: Effect of rhamnazin pretreatment on Nrf2 and Trx1 expression in lung tissues. The protein expression levels were determined as (A) western blot analysis (B) Densitometry of western blot was performed to estimate the relative protein concentration. Data are presented as the mean \pm standard error $(\mathrm{n}=10)$. $* \mathrm{P}<0.05$ compared with the LPS-induced ALI group; \#P $<0.05$ compared with the vehicle-treated ALI group. Nrf2, nuclear factor erythroid-2-related factor 2; Trx1, thioredoxin isoform 1. 
Wu et al., Afr J Tradit Complement Altern Med., (2017) 14 (4): 201-212

https://doi.org/10.21010/ajtcam.v14i4.23.

\section{Discussion}

In the present study, we identified the anti-inflammatory and antioxidative effects of rhamnazin on the LPSinduced ALI in rats. This study also attempts to determine the potential mechanism of action of rhamnazin on pulmonary inflammation. This is the first such study on rhamnazin, a natural flavonoid found in several plants. Results showed a significant attenuation of lung inflammation which was evident from parameters such as decreased wet-to-dry weight ratio of the lungs, the concentration of BALF protein, the activity of MPO, cytokine production and migration of inflammatory cells. Moreover, the expression of Nrf2 and Trx1 were also found to be significantly increased. Moreover, in all the treatment groups a significantly large decrease in the levels of $\mathrm{H}_{2} \mathrm{O}_{2}, \mathrm{MDA}$ and hydroxyl ion was observed.

The pathology of ALI in humans has strong similarities with the progression of LPS induced ALI in mouse model (Rojas et al; 2005). Thus, several researchers have found this model to be the most appropriate for the study of therapeutic agents. It has been shown that in LPS induced ALI, there is a significant increase in the levels of inflammatory cytokines, proteins, neutrophils in serum and BALF (Jerala, 2007; Lu et al; 2008). The widespread destruction of alveolar spaces, edema and infiltration of neutrophils in the alveolar cavities are also the hallmarks of ALI (Ware and Matthay, 2000;Piantadosi and Schwartz, 2004; Matthay and Zemans, 2011). Increase in the wet-to-dry weight ratio of the lungs (Jacob et al; 2008) and an elevated BALF protein concentration due to protein extravasation (Muller-Redetzky et al; 2014) are strong indicators of pulmonary inflammation and edema. Both of these parameters were significantly increased upon LPS-induction and there was a subsequent significant decrease after rhamnazin treatment. These results indicated attenuation of pulmonary edema and vascular leakage in LPS-challenged rats after rhamnazin treatment.

Pulmonary edema indicated by the wet-to-dry ratio of lungs as well as the BALF protein concentration was further confirmed by the observations in lung histology. We observed several inflammatory changes in our rat ALI-model for example, pulmonary edema, thickening of pulmonary epithelium and migration of the inflammatory cells into the pulmonary interstitial spaces. A marked improvement in the histopathology of lungs was noticed when the LPS-induced rats were treated with rhamnazin. This observation provided a direct proof of the effectiveness of rhamnazin in the LPSinduced ALI, apart from the various measured parameters.

Uncontrolled and sustained lung inflammation is known to play an important role in the pathogenesis of ALI (Goodman et al; 2003; Manicone, 2009). Therefore, a strategy for attenuation of ALI progression should preferably involve suppression of immune system mediated inflammation. In the present study we found that rhamnazin exerts a strong antiinflammatory response in LPS induced ALI. The levels of inflammatory cytokines were significantly reduced in the serum ad BALF of rhamnazin treated group as compared to untreated group. These results provide a strong indication that rhamnazin caused attenuation of LPS induced ALI by inhibition of inflammatory cytokines.

Neutrophil infiltration into lungs is one of the hallmarks of ALI (Lucas et al; 2009; Matthay and Zemans, 2011). After LPS-induction, neutrophils migrate across the pulmonary endothelium and epithelium and reach the alveolar spaces. Here, the activated neutrophils cause an elevated production of ROS, the release of MPO, cytotoxic and pro-inflammatory mediators and increase in micro-vascular permeability (Chignard and Balloy, 2000; Grommes and Soehnlein, 2011). Therefore, the persistent presence of neutrophils in the pulmonary cavity is a strong indicator of ALI. In this study, upon rhamnazin treatment, the total cell count in BALF as well as that of neutrophils and macrophages was significantly decreased. Neutrophils and alveolar macrophages are known to release MPO during acute inflammation of the lungs (Chagnon et al; 2015). Increased activity of MPO is a strong indicator of neutrophil migration into the pulmonary parenchyma and the alveolar cavities (Reumaux et al; 2003). MPO concentration has been used for estimation of LPSinduced ALI in many studies, for example,Rittirsch et al. (2008), Tsai et al. (2014). In the rhamnazin pretreated rats, a marked reduction in the activity of MPO was observed. This further confirmed a reduced neutrophil infiltration and diminished inflammation of lungs.

ROS are produced by activated cytokines (Tasoulis et al; 2009). ROS can cause several damages to lung tissue such as DNA damage, protein oxidation, and lipid peroxidation. In addition to being an anti-inflammatory agent, rhamnazin is also known to possess antioxidant properties (Martini et al; 2004). We observed heightened levels of molecules such as MDS, $\mathrm{H}_{2} \mathrm{O}_{2}$ and hydroxyl ion in the LPS-induced ALI in our results. These levels were significantly normalized with rhamnazin pre-treatment, further suggesting the efficacy of rhamnazin in the treatment of ALI. Nrf2 is a well known regulator of cellular antioxidant response (Wang et al; 2012). Additionally, the protein Trx 1 functions as a redox protein against conditions of oxidative stress (Furukawa et al; 2011). In this study, we found that the levels of Nrf2 and Trx1 were significantly deactivated in LPS induced ALI animals. However, a significant up-regulation of Nrf2 and Trx1 with rhamnazin pretreatment further supported its strong antioxidant properties.

In this study, plant flavonoid, rhamnazin showed protective properties against LPS-induced ALI. All the parameters for measuring pulmonary inflammation showed a significant improvement over the LPS lung. These protective 
Wu et al., Afr J Tradit Complement Altern Med., (2017) 14 (4): 201-212

https://doi.org/10.21010/ajtcam.v14i4.23.

properties may be partly due to the inhibition of inflammatory cytokines production. Moreover, rhamnazin may also function by its strong antioxidant properties. The strong antioxidant properties of rhamnazin are associated with its ability to activate the Nrf2 pathway. The strong antioxidant properties of rhamnazin found $\mathrm{n}$ this study and reported earlier.

\section{Acknowledgement}

This study was funded by institutional research grant of Changzhou jintan district people's hospital

\section{References}

1. Cachofeiro, V., M. Goicochea, S. G. de Vinuesa, P. Oubina, V. Lahera, and J. Luno. 2008. Oxidative stress and inflammation, a link between chronic kidney disease and cardiovascular disease. Kidney Int Suppl:S4-9.

2. Chagnon, F., A. Bourgouin, R. Lebel, M. A. Bonin, E. Marsault, M. Lepage, and O. Lesur. 2015. Smart imaging of acute lung injury: exploration of myeloperoxidase activity using in vivo endoscopic confocal fluorescence microscopy. Am. J. Physiol. Lung Cell Mol. Physiol. 309:L543-551.

3. Chignard, M. and V. Balloy. 2000. Neutrophil recruitment and increased permeability during acute lung injury induced by lipopolysaccharide. Am. J. Physiol. Lung Cell Mol. Physiol. 279:L1083-1090.

4. Deramaudt, T. B., C. Dill, and M. Bonay. 2013. Regulation of oxidative stress by Nrf2 in the pathophysiology of infectious diseases. Med Mal Infect 43:100-107.

5. Fouad, A. A., W. H. Albuali, and I. Jresat. 2016. Protective Effect of Naringenin against Lipopolysaccharide-Induced Acute Lung Injury in Rats. Pharmacology 97:224-232.

6. Frutos-Vivar, F., N. Nin, and A. Esteban. 2004. Epidemiology of acute lung injury and acute respiratory distress syndrome. Curr. Opin. Crit. Care 10:1-6.

7. Furukawa, M., R. Tanaka, V. T. Chuang, Y. Ishima, K. Taguchi, H. Watanabe, T. Maruyama, and M. Otagiri. 2011. Human serum albumin-thioredoxin fusion protein with long blood retention property is effective in suppressing lung injury. $\mathrm{J}$ Control Release 154:189-195.

8. Goodman, R. B., J. Pugin, J. S. Lee, and M. A. Matthay. 2003. Cytokine-mediated inflammation in acute lung injury. Cytokine Growth Factor Rev 14:523-535.

9. Grommes, J. and O. Soehnlein. 2011. Contribution of neutrophils to acute lung injury. Mol. Med. 17:293-307.

10. Huang, R., T. Zhong, and H. Wu. 2015. Quercetin protects against lipopolysaccharide-induced acute lung injury in rats through suppression of inflammation and oxidative stress. Arch. Med. Sci. 11:427-432.

11. Ingenito, E. P., R. Mora, M. Cullivan, Y. Marzan, K. Haley, L. Mark, and L. A. Sonna. 2001. Decreased surfactant proteinB expression and surfactant dysfunction in a murine model of acute lung injury. Am. J. Respir. Cell Mol. Biol. 25:35-44.

12. Jacob, S., D. J. Deyo, R. A. Cox, D. L. Traber, and H. K. Hawkins. 2008. Assessment of lung inflammation in a mouse model of smoke inhalation and burn injury: strain-specific differences. Toxicol. Mech. Methods 18:551-559.

13. Jerala, R. 2007. Structural biology of the LPS recognition. Int J Med Microbiol 297:353-363.

14. Karmpaliotis, D., I. Kosmidou, E. P. Ingenito, K. Hong, A. Malhotra, M. E. Sunday, and K. J. Haley. 2002. Angiogenic growth factors in the pathophysiology of a murine model of acute lung injury. Am. J. Physiol. Lung Cell Mol. Physiol. 283:585-595.

15. Kim, K., Y. Li, G. Jin, W. Chong, B. Liu, J. Lu, K. Lee, M. Demoya, G. C. Velmahos, and H. B. Alam. 2012. Effect of valproic acid on acute lung injury in a rodent model of intestinal ischemia reperfusion. Resuscitation 83:243-248.

16. Lee, H. S., P. Kang, K. Y. Kim, and G. H. Seol. 2015. Foeniculum vulgare Mill. Protects against Lipopolysaccharideinduced Acute Lung Injury in Mice through ERK-dependent NF-kappaB Activation. Korean J Physiol Pharmacol 19:183189.

17. Lee, J. P., Y. C. Li, H. Y. Chen, R. H. Lin, S. S. Huang, H. L. Chen, P. C. Kuan, M. F. Liao, C. J. Chen, and Y. H. Kuan. 2010. Protective effects of luteolin against lipopolysaccharide-induced acute lung injury involves inhibition of MEK/ERK and PI3K/Akt pathways in neutrophils. Acta Pharmacol Sin 31:831-838.

18. Li, G., C. L. Zhou, Q. S. Zhou, and H. D. Zou. 2016. Galantamine protects against lipopolysaccharide-induced acute lung injury in rats. Braz J Med Biol Res 49:e5008.

19. Li, Q. and I. M. Verma. 2002. NF-kappaB regulation in the immune system. Nat. Rev. Immunol. 2:725-734.

20. Liu, J. and A. Lin. 2007. Wiring the cell signaling circuitry by the NF-kappa B and JNK1 crosstalk and its applications in human diseases. Oncogene 26:3267-3278.

21. Livak, K. J. and T. D. Schmittgen. 2001. Analysis of relative gene expression data using real-time quantitative PCR and the $2^{\text {-DD CT }}$ Method. Methods 25:402-408. 
Wu et al., Afr J Tradit Complement Altern Med., (2017) 14 (4): 201-212

https://doi.org/10.21010/ajtcam.v14i4.23.

22. Lu, Y. C., W. C. Yeh, and P. S. Ohashi. 2008. LPS/TLR4 signal transduction pathway. Cytokine 42:145-151.

23. Lucas, R., A. D. Verin, S. M. Black, and J. D. Catravas. 2009. Regulators of endothelial and epithelial barrier integrity and function in acute lung injury. Biochem. Pharmacol. 77:1763-1772.

24. Luh, S. P. and C. H. Chiang. 2007. Acute lung injury/acute respiratory distress syndrome (ALI/ARDS): the mechanism, present strategies and future perspectives of therapies. J Zhejiang Univ Sci B 8:60-69.

25. Manicone, A. M. 2009. Role of the pulmonary epithelium and inflammatory signals in acute lung injury. Expert Rev Clin Immunol 5:63-75.

26. Martini, N. D., D. R. Katerere, and J. N. Eloff. 2004. Biological activity of five antibacterial flavonoids from Combretum erythrophyllum (Combretaceae). J Ethnopharmacol 93:207-212.

27. Matthay, M. A. and R. L. Zemans. 2011. The acute respiratory distress syndrome: pathogenesis and treatment. Annu. Rev. Pathol. 6:147.

28. Muller-Redetzky, H. C., N. Suttorp, and M. Witzenrath. 2014. Dynamics of pulmonary endothelial barrier function in acute inflammation: mechanisms and therapeutic perspectives. Cell Tissue Res. 355:657-673.

29. Nijveldt, R. J., E. van Nood, D. E. van Hoorn, P. G. Boelens, K. van Norren, and P. A. van Leeuwen. 2001. Flavonoids: a review of probable mechanisms of action and potential applications. Am. J. Clin. Nutr. 74:418-425.

30. Pedruzzi, L. M., M. B. Stockler-Pinto, M. Leite, Jr., and D. Mafra. 2012. Nrf2-keap1 system versus NF-kappaB: the good and the evil in chronic kidney disease? Biochimie 94:2461-2466.

31. Piantadosi, C. A. and D. A. Schwartz. 2004. The acute respiratory distress syndrome. Ann. Intern. Med. 141:460-470.

32. Reumaux, D., M. de Boer, A. B. Meijer, P. Duthilleul, and D. Roos. 2003. Expression of myeloperoxidase (MPO) by neutrophils is necessary for their activation by anti-neutrophil cytoplasm autoantibodies (ANCA) against MPO. J. Leukoc. Biol. 73:841-849.

33. Rittirsch, D., M. A. Flierl, D. E. Day, B. A. Nadeau, S. R. McGuire, L. M. Hoesel, K. Ipaktchi, F. S. Zetoune, J. V. Sarma, L. Leng, M. S. Huber-Lang, T. A. Neff, R. Bucala, and P. A. Ward. 2008. Acute lung injury induced by lipopolysaccharide is independent of complement activation. J. Immunol. 180:7664-7672.

34. Rojas, M., C. R. Woods, A. L. Mora, J. Xu, and K. L. Brigham. 2005. Endotoxin-induced lung injury in mice: structural, functional, and biochemical responses. Am J Physiol Lung Cell Mol Physiol 288:L333-341.

35. Sanlioglu, S., C. M. Williams, L. Samavati, N. S. Butler, G. Wang, P. B. McCray, Jr., T. C. Ritchie, G. W. Hunninghake, E. Zandi, and J. F. Engelhardt. 2001. Lipopolysaccharide induces Rac1-dependent reactive oxygen species formation and coordinates tumor necrosis factor-alpha secretion through IKK regulation of NF-kappa B. J. Biol. Chem. 276:30188-30198.

36. Sato, K., M. B. Kadiiska, A. J. Ghio, J. Corbett, Y. C. Fann, S. M. Holland, R. G. Thurman, and R. P. Mason. 2002. In vivo lipid-derived free radical formation by NADPH oxidase in acute lung injury induced by lipopolysaccharide: a model for ARDS. FASEB J. 16:1713-1720.

37. Suliman, H. B., M. S. Carraway, and C. A. Piantadosi. 2003. Postlipopolysaccharide oxidative damage of mitochondrial DNA. Am. J. Respir. Crit. Care Med. 167:570-579.

38. Tang, G., Y. Minemoto, B. Dibling, N. H. Purcell, Z. Li, M. Karin, and A. Lin. 2001. Inhibition of JNK activation through NF-kappaB target genes. Nature 414:313-317.

39. Tasoulis, M. K., O. Livaditi, M. Stamatakos, C. Stefanaki, P. Paneris, P. Prigouris, A. Flevari, N. Goutas, D. Vlachodimitropoulos, V. Villiotou, and E. E. Douzinas. 2009. High concentrations of reactive oxygen species in the BAL fluid are correlated with lung injury in rabbits after hemorrhagic shock and resuscitation. Tohoku J. Exp. Med. 219:193199.

40. Trocha, M., A. Merwid-Lad, E. Chlebda, T. Sozanski, M. Piesniewska, H. Gliniak, and A. Szelag. 2014. Influence of ezetimibe on selected parameters of oxidative stress in rat liver subjected to ischemia/reperfusion. Arch Med Sci 10:817824.

41. Tsai, C. L., Y. C. Lin, H. M. Wang, and T. C. Chou. 2014. Baicalein, an active component of Scutellaria baicalensis, protects against lipopolysaccharide-induced acute lung injury in rats. J. Ethnopharmacol. 153:197-206.

42. Wang, B., X. Zhu, Y. Kim, J. Li, S. Huang, S. Saleem, R. C. Li, Y. Xu, S. Dore, and W. Cao. 2012. Histone deacetylase inhibition activates transcription factor Nrf2 and protects against cerebral ischemic damage. Free Radic Biol Med 52:928936.

43. Ware, L. B. and M. A. Matthay. 2000. The acute respiratory distress syndrome. N. Engl. J. Med. 342:1334-1349.

44. Yang, J., W. Li, M. Duan, Z. Zhou, N. Lin, Z. Wang, J. Sun, and J. Xu. 2005. Large dose ketamine inhibits lipopolysaccharide-induced acute lung injury in rats. Inflamm Res 54:133-137.

45. Ye, J., G. Coulouris, I. Zaretskaya, I. Cutcutache, S. Rozen, and T. L. Madden. 2012. Primer-BLAST: a tool to design target-specific primers for polymerase chain reaction. BMC Bioinformatics 13:134. 